\title{
Disruption Mitigation Efficiency and Scaling with Thermal Energy Fraction on ASDEX Upgrade
}

\author{
U. A. Sheikh ${ }^{1}$, P. David ${ }^{2}$, O. Ficker ${ }^{3}$, M. Bernert ${ }^{2}$, D. Brida ${ }^{2}$, \\ M. Dibon ${ }^{2}$, B. Duval ${ }^{1}$, M. Faitsch ${ }^{2}$, M. Maraschek ${ }^{2}$, G. Papp ${ }^{2}$, \\ G. Pautasso ${ }^{2}$, C. Sozzi ${ }^{4}$, the AUG team ${ }^{5}$ and EUROfusion \\ MST1 team ${ }^{6}$ \\ ${ }^{1}$ École Polytechnique Fédérale de Lausanne (EPFL), Swiss Plasma Center (SPC), \\ CH-1015 Lausanne, Switzerland \\ ${ }^{2}$ Max Planck Institute für Plasmaphysik, Garching, Germany \\ ${ }^{3}$ Institute of Plasma Physics AS CR, Za Slovankou 1782/3, 18200 Praha 8, Czech \\ Republic \\ ${ }^{4}$ Istituto di Fisica del Plasma - Universita degli Studi di Milano-Bicocca, Milan, Italy \\ ${ }^{5}$ See the author list Meyer, H. et al. Nucl. Fusion 59 (2019) 112014 \\ ${ }^{6}$ See the author list Labit, B. et al Nucl. Fusion 59 (2019) 086020 \\ E-mail: umar.sheikh@epfl.ch
}

August 2020

\begin{abstract}
Disruption mitigation remains a critical and unresolved issue for ITER. Measurement uncertainties preventing quantification of a system's efficacy remains a significant hurdle in producing and validating a viable disruption mitigation system. This study addresses this issue through the creation of a dataset on the ASDEX Upgrade tokamak targeted at developing analysis techniques to quantify mitigation that are applicable irrespective of the disruption mitigation system being studied. These experiments used a range of thermal energy fractions to address concerns for ITER from JET massive gas injection (MGI) experiments showing a decrease in efficacy with increasing thermal energy fraction. The dataset produced in this study used MGI valves in two toroidal locations. The high resolution foil bolometers and AXUV diode arrays were used to infer radiation emission profiles at varying toroidal distances from the injection location. The fueling efficiency of the two systems was found to be comparable and toroidal asymmetries over the entire disruption were found to be negligible. The AXUV diodes were cross-calibrated with the foil bolometers and used to estimate the thermal energy radiated and magnetic energy coupled to the vessel structure and coils. It was estimated that $75-95 \%$ of the thermal energy was radiated and an almost constant $60 \%$ of the total magnetic energy was coupled. Radiated energy fractions of 0.8-1.0 were calculated and no decrease as a function of thermal energy fraction was found. A $\pm 20 \%$ variation in the coupled magnetic energy was explored and it did not alter this trend.
\end{abstract}

Keywords: Disruption mitigation, power balance, bolometers 


\section{Introduction}

Disruptions have the potential to release large amounts of thermal and magnetic energy ( $W_{\text {th }}$ and $W_{\text {mag }}$ ) in millisecond time-scales and this can result in extreme heat loads on plasma facing components and electromagnetic (EM) forces on the vessel [1]. Existing machines are capable of handling these forces and loads as they were designed to study the disruptive boundaries of tokamak operation. It is not technically feasible to allow unmitigated disruptions at the high stored energies on ITER as the predicted heat load would evaporate up to $100 \mu \mathrm{m}$ of the divertor target plate [2]. The last line of defence to prevent such an outcome is a disruption mitigation system. The most common approach is injection of a massive amount of material relative to the plasma mass. The goal of such a system is threefold:

- Radiation of $W_{\text {th }}$ before it can be deposited on the targets

- Mitigation of EM loads

- Prevention or mitigation of high current "runaway" electron beams

Specifically, the ITER mitigation system needs to be able to reduce vessel forces by a factor of 2-3 and the thermal loads by at least $90 \%$ to ensure the integrity and lifetime of the machine $[3,4]$. Runaway electrons (RE) are of particular concern on ITER as they are expected to exceed 10 MA [1]. Relatively low RE currents of 0.5 MA on JET have caused average temperature increases of up to $700^{\circ} \mathrm{C}$ on the upper dump plate and concentrated localised damage on impact with a wall $[1,5]$. Thus a RE current of $10 \mathrm{MA}$ would cause severe melting of plasma facing components on ITER and must be avoided. As of yet, no technique has been proven to reliably achieve these targets for a range of plasma scenarios.

Two main systems for massive material injection have been extensively studied; massive gas injection (MGI) and shattered pellet injection (SPI). MGI has been explored on a range of tokamaks including Alcator C-mod, ASDEX Upgrade (AUG), DIII-D, JT60-U, MAST, TEXTOR, Tore Supra, J-TEXT and HL-2A [6, 7, 8, 9, 10, 11, 12]. SPI on the other hand is relatively new and has only been studied in detail on DIII-D with further, more recent experiments on JET, K-STAR, J-TEXT and HL$2 \mathrm{~A}[13,14,15,16,17]$. MGI and SPI systems have reported over $90 \%$ of the initial $W_{\text {mag }}$ as being radiated $[18,19]$, however the scatter on total radiated energy $\left(W_{\text {rad }}\right)$ is large depending on machine parameters as shown in Figure 1 [20].

A particularly worrying trend observed on JET has been the decrease in radiated energy fraction $\left(f_{\mathrm{rad}}\right)$ with increasing stored thermal energy fraction $\left(f_{\mathrm{th}}\right)$, as shown in Figure $2[20,21,22]$. The definition used in this study for the two parameters is outlined in Equations 1 and 2. $W_{\text {th }}$ denotes the stored thermal energy, $W_{\text {mag }}$ the stored magnetic energy and $W_{\text {coupled }}$ the magnetic energy dissipated in the surrounding structure (vessel and coils) during the current quench (CQ). This has been calculated for the dataset presented in Figure 2 using the circuit model, similar to that described in Lehnen 2013 [23]. The $W_{\text {rad }}$ presented has been inferred using the horizontal foil bolometer 


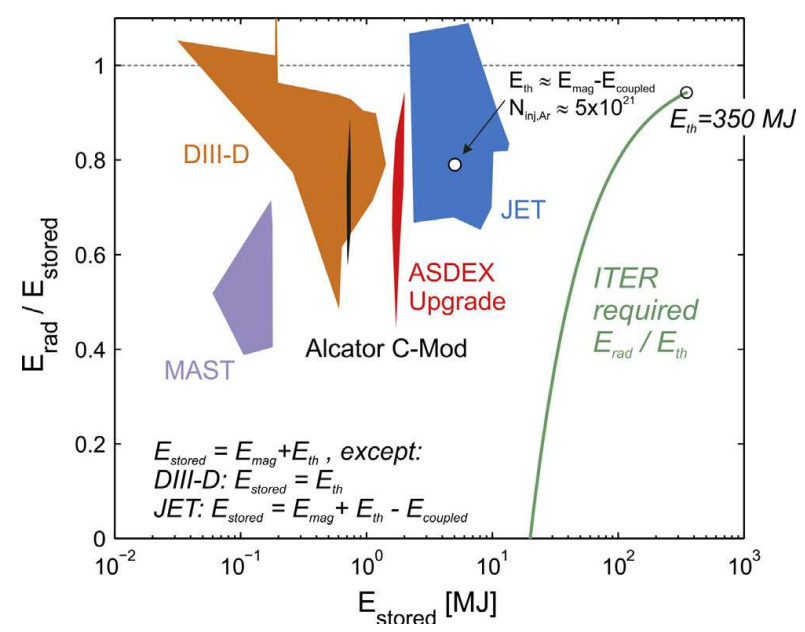

Figure 1. MGI efficiency using high- $Z$ noble gases (neon, argon) and mixtures of these with $\mathrm{D}_{2}$ and He. The white JET data point indicates the maximum achievable radiated energy fraction at high thermal energy fraction on JET [20].

array and integrated across the entire disruption. This trend is extremely concerning as ITER is targeting to operate close to an $f_{\text {th }}$ of 0.6 during high performance discharges.

$$
\begin{aligned}
& f_{\mathrm{rad}}=W_{\mathrm{rad}} /\left(W_{\mathrm{mag}}+W_{\mathrm{th}}-W_{\text {coupled }}\right) \\
& f_{\mathrm{th}}=W_{\mathrm{th}} /\left(W_{\mathrm{mag}}+W_{\mathrm{th}}-W_{\text {coupled }}\right)
\end{aligned}
$$

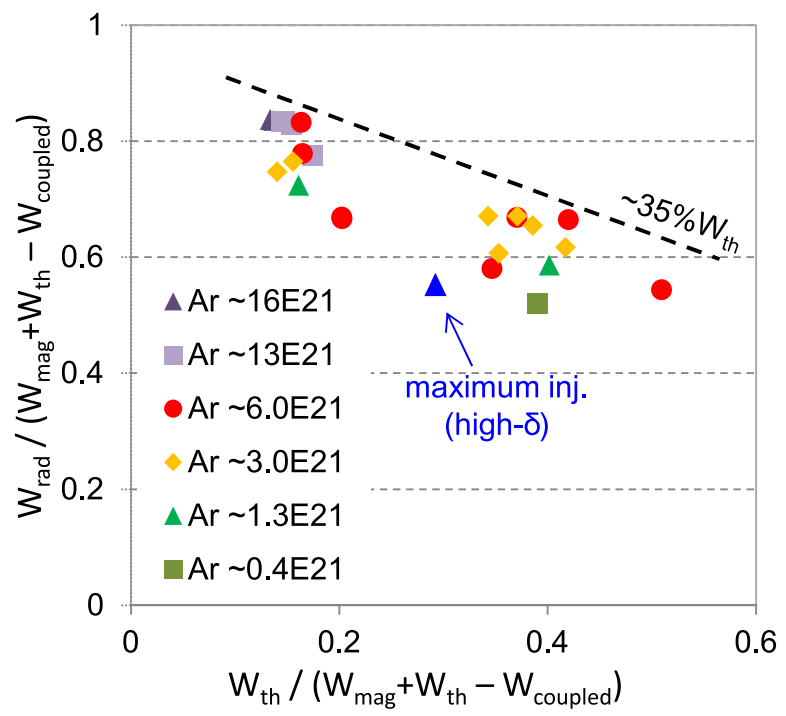

Figure 2. Reduction of $f_{\text {rad }}$ with increasing $f_{\text {th }}$ observed with JET MGI [21].

The inferred mitigation efficiency for similar discharges can have large variations and uncertainties as measurements using the standard set of diagnostics for $W_{\text {rad }}$ and heat fluxes on plasma facing components can be severely hampered during a mitigated disruption. For example, foil bolometers that are used to measure absolute radiated 
power operate on time scales too slow to infer $W_{\text {rad }}$ before the thermal quench (TQ). AXUV diodes are fast enough to separate the TQ and CQ but if they are slightly aged, their sensitivity can vary as a function of wavelength. However, it has been shown that the diode response does settle after roughly 300 seconds of plasma operation [24].

This makes the diodes unsuitable for direct absolute measurements of $W_{\text {rad. }}$. Heat flux measurements using infra-red cameras can be difficult due to IR emission from the injected gas and reflections from the vessel structure producing background signal and artefacts that taint the calibration. Experiments on carbon wall machines such as DIIID have been able to use post disruption tile temperatures to infer information about the heat loads. This was possible due to the lower thermal conductivity of carbon resulting in a hotter tile surface for a longer period of time after the disruption. Such an approach is more difficult on metal wall machines due to higher thermal conductivity, lower emissivity and higher reflectivity [25].

These diagnostic challenges result in an uncertainty that is larger than the mitigation efficiency criteria. Without a reduction in these uncertainties it is currently not possible to ensure a mitigation system can meet requirements on a larger machine such as ITER. To this end, the goals of this study are to experimentally investigate the reduction in $f_{\text {rad }}$ with increasing $f_{\text {th }}$ on $\mathrm{AUG}$, investigate the impact of inferred $W_{\text {rad }}$ as a function of toroidal distance between injection and measurement location and estimate the uncertainties in the energy balance methodology. It is envisioned that the diagnostic analysis developed in this study be applicable independent of the material injection system being employed.

\section{Experiment Description and Diagnostics}

AUG is a D shaped full metal wall machine with a major radius of $1.65 \mathrm{~m}$ and field and plasma current of up to 3.2 T and 1.4 MA respectively [26]. The auxiliary heating systems are capable of providing up to $20 \mathrm{MW}$ of neutral beam heating (NBH), $7 \mathrm{MW}$ of ion cyclotron heating (ICRH) and $6 \mathrm{MW}$ of electron cyclotron heating (ECRH) split between $140 \mathrm{GHz}$ and $105 \mathrm{GHz}[27]$. AUG is uniquely equipped with MGI systems in several toroidal and poloidal locations as well as a large range of relevant diagnostics including a full array of foil bolometers in a single toroidal location, AXUV diode arrays in two toroidal locations, infra-red (IR) cameras, fast CO2 interferometers and strain gauges on the vessel support structure [28].

Figure 3c illustrates the toroidal positions of the primary diagnostics and gas injection systems used in this study. The blue rectangles represent the three IR cameras that were used in this study and their relative viewing location. These were arranged to provide a wide view of the overall divertor, two tiles on the outer target optimised for IR measurements and an optimised "strip" on the outer target. The electromagnetic (EM) loads were monitored using strain gauges on the support columns of the tokamak and shunt resistors in the divertor to estimate Halo currents $\left(I_{\text {halo }}\right)$. 


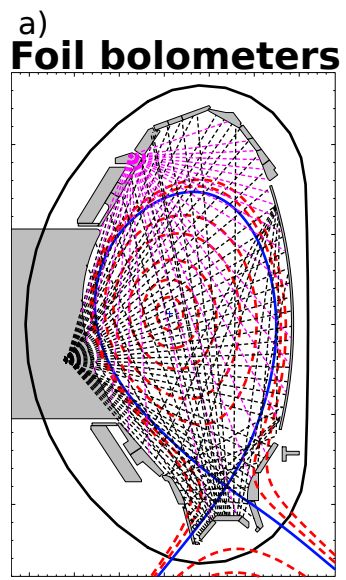

b)

AXUV diodes

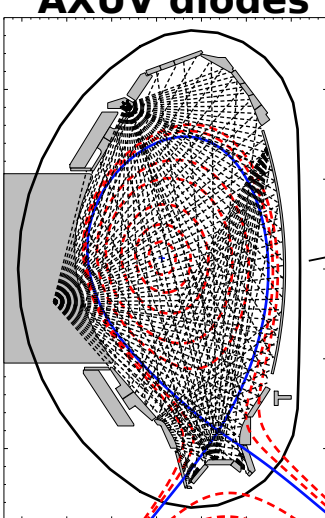

c)

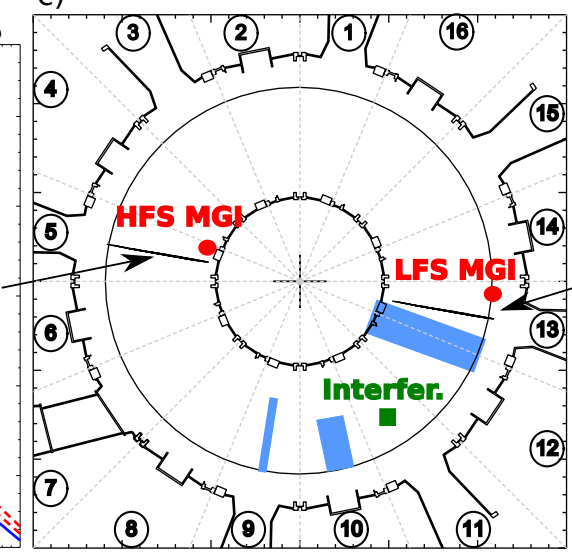

d) AXUV diodes

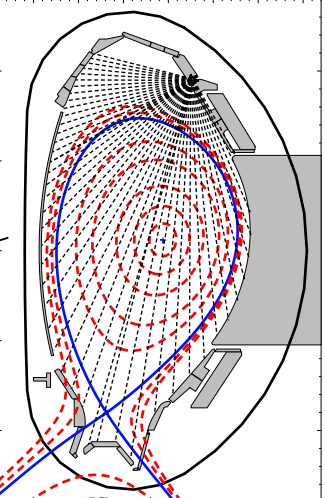

Figure 3. Poloidal lines of sight of the foil bolometers (a), AXUV diodes in sector 5 (b) and in sector 13 (d) and their relative toroidal locations (c). Blue rectangles locations and approximate viewing regions of the IR cameras. Red circles - locations of the two MGI systems. Green rectangle - vertical line of sight of the CO2 interferometer.

\subsection{Massive Gas Injection Systems}

The MGI valves used in this study were in-vessel on the low field side (LFS) and high field side (HFS) in sectors 13 and 5 respectively. Both valves are capable of opening in approximately $1 \mathrm{~ms}$ and withstanding the demanding in-vessel environment. They operate using a pneumatic piston driven by compressed air, pairs of piezoelectricactuators and a disk spring that opens the valves as outlined in Pautasso 2008 and $2015[28,29]$.

The MGI systems were operated with a mixture of $90 \%$ deuterium and $10 \%$ neon or argon. The maximum allowed quantity limited by deuterium use regulations, was used for the "large" injection discharges with $\sim 1.7 \cdot 10^{22}$ particles injected. "Small" injections of $\sim 0.9 \cdot 10^{22}$ particles were also performed to investigate whether $f_{\text {rad }}$ had saturated to have a direct comparison with JET results.

\subsection{Radiation Diagnostics}

Foil bolometers and AXUV diodes are used during standard operation on AUG over a range of poloidal locations to achieve full coverage in sector 5. An additional AXUV camera on the upper LFS is also available in sector 13 providing a single "fan" of chords across the machine. The operation of these diagnostics and the analysis techniques compared are briefly introduced in the following subsections.

\subsubsection{Foil Bolometers}

Foil bolometers provide absolute radiation measurements due to their flat spectral response from approximately $50 \mathrm{eV}$ to the $\mathrm{keV}$ range [30]. The temporal resolution during standard operation is $30 \mathrm{~ms}$ and is a result of their thermal inertia as they operate, essentially, as thermistor thermometers. The high radiative power achieved 
with MGI in this study resulted in higher heat fluxes on the foils, producing larger bridge voltages, allowing a reduction in the temporal averaging window to $400 \mu \mathrm{s}$ whilst maintaining signal to noise ratios comparable to standard operation.

The bolometery system on AUG consists of 112 lines from 6 cameras as shown in Figure 3a. All six cameras were analysed with two tomographic inversion techniques to provide radiated power as a function of time. The standard tomographic inversion technique on AUG allows spatial smoothing along flux surfaces (Flux Surface Smoothing - FSS) and does not reject negative values [31]. The drawback of this approach is a valid equilibrium reconstruction is required and this is often challenging during a disruption. The second inversion technique requires no equilibrium information, instead resolving the power density on a rectangular grid after smoothing the experimental data in the $R$ and $z$ directions [32]. This technique does not permit negative power values. These two techniques will be referred to as "FSS" and "no FSS" respectively.

A third radiated power inference technique was applied using a single camera indicated using magenta lines of sight in Figure 3a. Here, constant radiation along flux surfaces is assumed and does not produce inverted images [33]. This method provides a direct comparison with JET measurements, which uses only a single camera. This technique will be referred to as "single camera" for this study.

\subsubsection{AXUV Diode Arrays}

AXUV diodes are silicon based detectors that are only sensitive to incoming photons with a response that is a function of photon energy. This sensitivity variation can be exacerbated over time by damage from high energy photons resulting in significantly reduced sensitivity in the ultra-violet (UV) spectral range, as shown in Figure 4[24]. Whilst it has been shown that their response stabilises after a given exposure time, they cannot be used directly for absolute measurements of radiated power. Their advantage is a high temporal resolution (up to $500 \mathrm{kHz}$ on $\mathrm{AUG}$ ) allowing qualitative studies of fast transient events such as disruption mitigation.

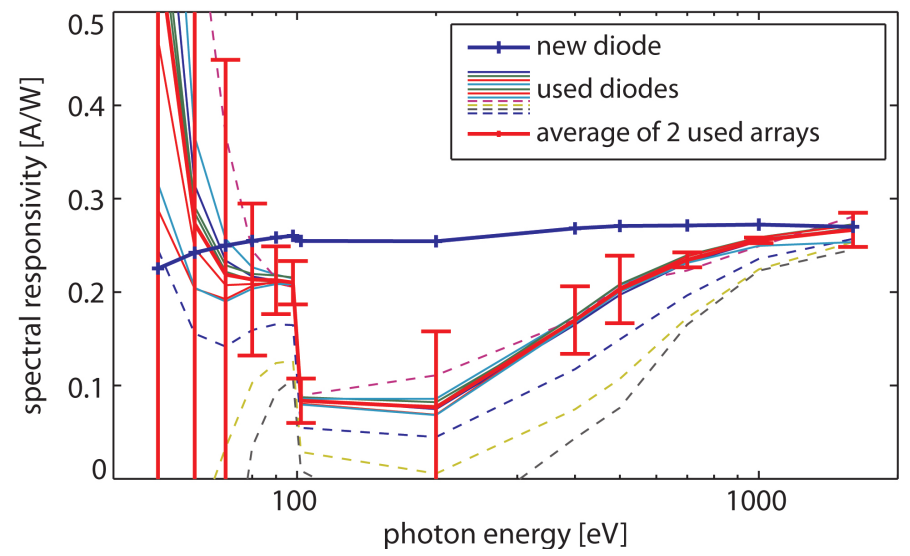

Figure 4. Spectral response of new and used AXUV diodes measured in a synchrotron [24] 
Table 1. Nominal plasma parameters used and heating employed for the high and low $f_{\text {th }}$ scenarios.

\begin{tabular}{ccc}
\hline$I_{\mathrm{p}}=0.8 \mathrm{MA}$ & $B_{\mathrm{T}}=2.5 \mathrm{~T}$ & $q_{95} 4.9$ \\
$\kappa=1.72$ & $\delta_{\mathrm{o}}=0.11$ & $\delta_{\mathrm{u}}=0.50$ \\
\hline High $W_{\text {th }}$ Scenario & $7.5 \mathrm{MW} \mathrm{NBH}$ & $3.5 \mathrm{MW} \mathrm{ECRH}$ \\
\hline Low $W_{\text {th }}$ Scenario & $2.5 \mathrm{MW} \mathrm{NBH}$ & $1.8 \mathrm{MW} \mathrm{ECRH}$ \\
\hline
\end{tabular}

The AXUV system on AUG consists of 176 lines of sight in sector 5 as shown by the black lines in Figure $3 \mathrm{~b}$ and foil bolometers in the same sector allow for crosscalibration. An additional single camera system with 32 lines of sight is available in sector 13, as shown in Figure 3d. Inversions using Pytomo [34] were performed for both systems independently allowing a qualitative comparison of radiation asymmetries. This inversion technique allows FSS and negative emission values.

\subsection{Scenario Description}

AUG was operated well below its performance limits to ensure machine safety during potentially poorly mitigated discharges. A single scenario was selected for the whole study with approximately $1 \mathrm{MJ}$ of $W_{\text {mag }}$. Auxiliary heating was varied to provide a scan in $W_{\text {th }}$ and consequently $f_{\text {th }}$. The scenario parameters with the heating schemes for "high" and "low" $W_{\text {th }}$ are provided in Table 1. Each reported discharge terminated following MGI injection into a healthy plasma at $3.000 \mathrm{~s}$ for LFS injection and $3.035 \mathrm{~s}$ for HFS injection.

Figure 5 provides an overview of a reference high power discharge (\#35653) mitigated with the LFS valve in sector 13 . The vertical lines indicate the nominal sequence of events; valve opening (red), gas arrival at plasma edge (green), start of the TQ (black) and start of CQ(magenta). The arrival of the gas at the plasma edge is inferred from the AXUV diodes in the injection sector. The time between gas arrival and the start of the TQ will be referred to as the pre-TQ time. The sharp drop in core soft x-ray (SXR) emission indicates the loss of $W_{\text {th }}$ during the TQ and the slight preceding increase in plasma current marks the beginning of the CQ.

\section{Results}

A dataset comprising of 11 discharges mitigated using the sector 13 LFS valve and 6 discharges mitigated using the sector 5 HFS valve were produced for this study. The following sections present analyses of MGI injection and assimilation, radiated energy and the mitigation of thermal and magnetic energy. The energy loss channels are then combined to generate an energy balance and compute $f_{\text {rad }}$. 

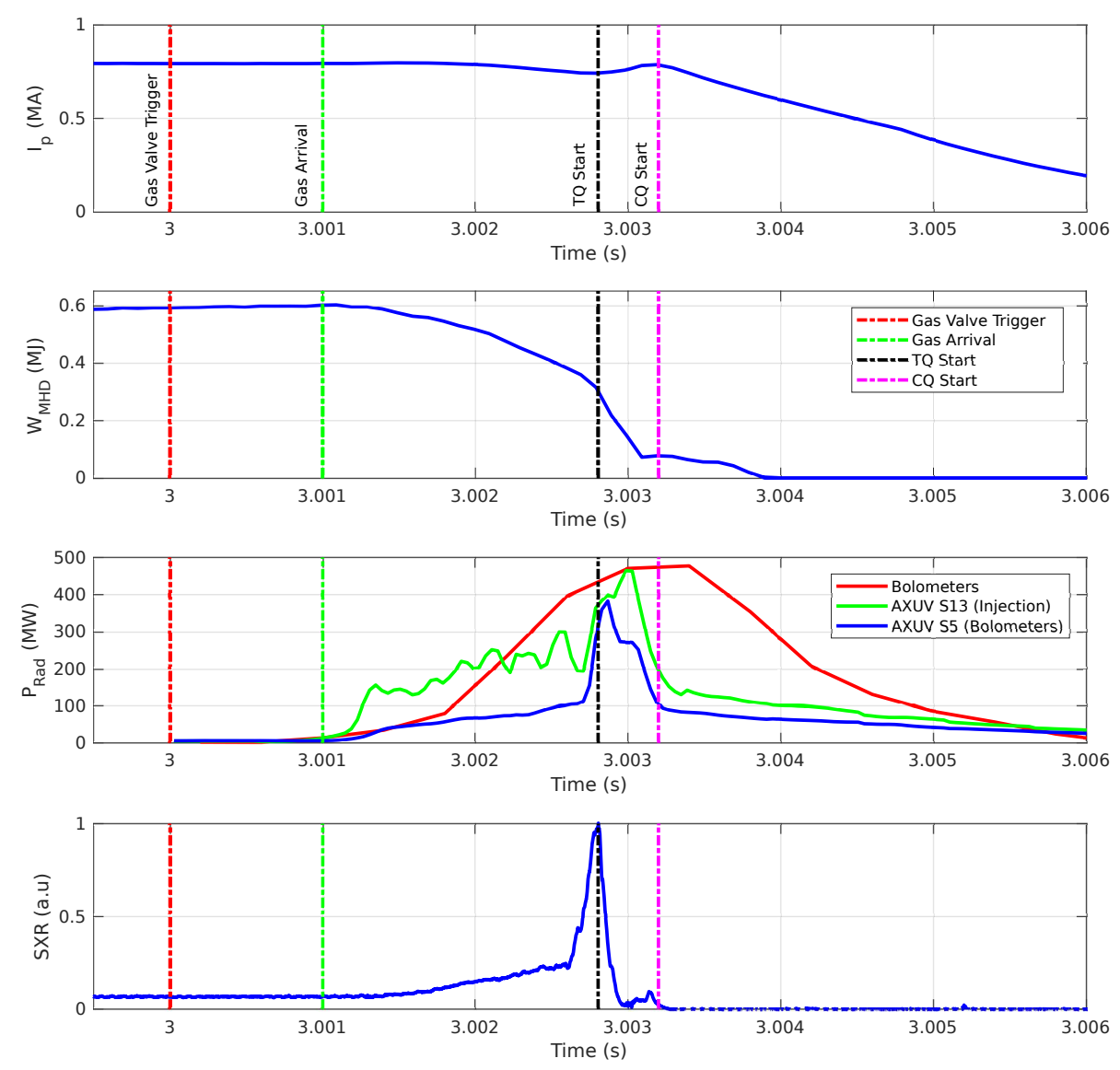

Figure 5. Nominal discharge plasma current, $W_{\text {th }}$, radiated power and core SXR emission from top to bottom. Dashed lines mark valve opening, gas arrival, TQ start and CQ start from left to right.

\subsection{MGI Injection and Assimilation}

The response time of the MGI valves can be estimated from the flight time of the injected gas; the delay between trigger time of the MGI and a doubling of the AXUV diodes signal in the injection sector. Figure 6 left presents the flight time for both valves using $10 \%$ argon and neon mixtures and two injection quantities. The number of injected particles was controlled by the pressure in the MGI reservoir. This required a lower gas pressure for the larger reservoir of the HFS valve to match the total number of particles injected. The flight time using the HFS valve was shorter than for the LFS valve due to valve position relative to the diodes. The neon mixture reached the plasma in less than one ms from the HFS valve and as expected, the argon mixture took longer.

The pre-TQ time as a function of injected particles is presented in Figure 6 right and it does not appear to be a function of gas mixture but does vary with valve location. Injections from the LFS valve, shown by empty symbols, appear to have an almost constant pre-TQ time of $1.5-1.8 \mathrm{~ms}$, whereas injections from the HFS valve have approximately double the pre-TQ time. This is attributed to two factors: a delay in detecting gas arrival for the LFS valve and a relatively lower reservoir pressure (due 

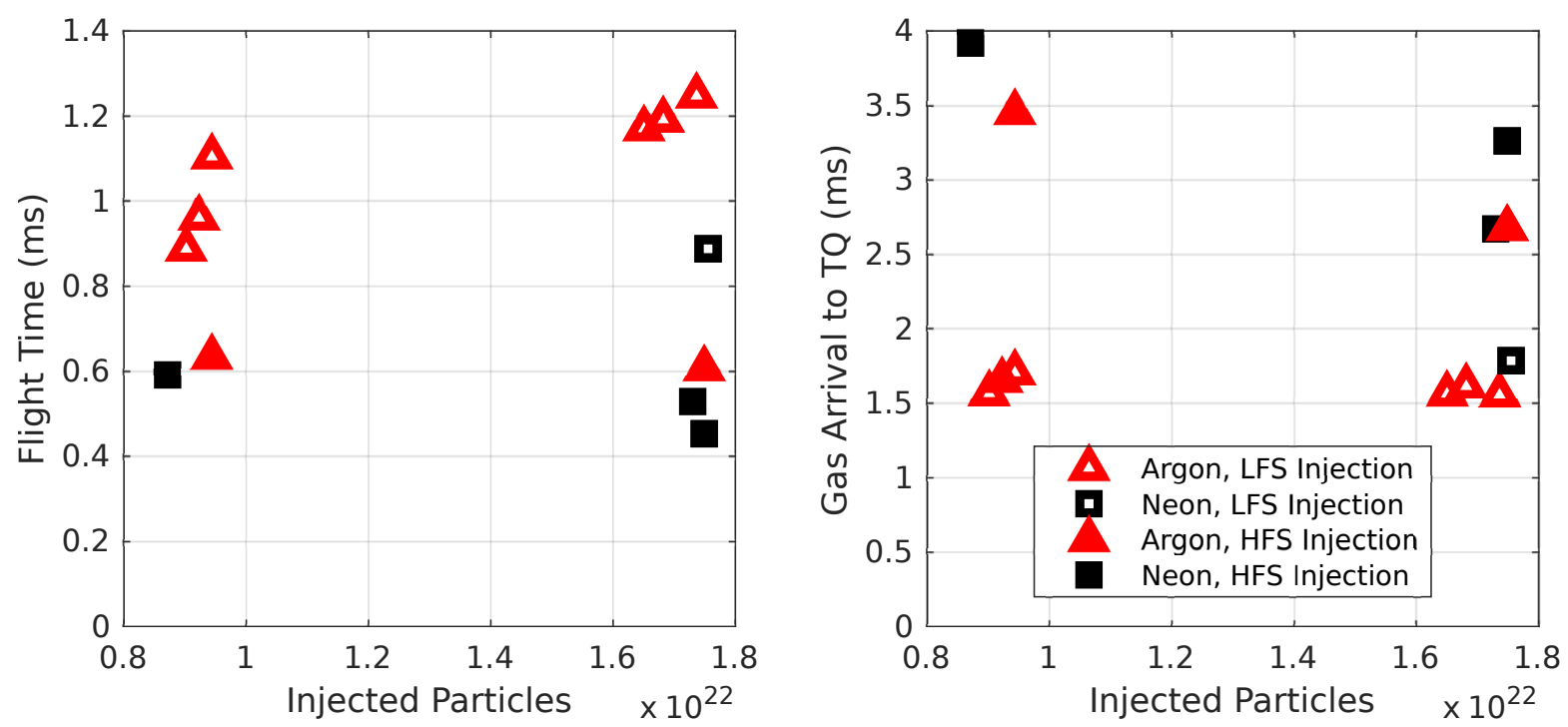

Figure 6. Flight time of particles (left) and pre-TQ time of each discharge as a function of number of particles injected (right).

to a larger volume) producing a lower flow rate for the HFS.

The quantity of gas assimilated with "large" injections could not be estimated from density measurements that were corrupted by numerous fringe jumps. The smaller injections produced fewer fringe jumps and density traces for two representative neon injections into high $W_{\text {th }}$ discharges are presented in Figure 7 . An earlier increase in density is observed for LFS injection ascribed to its toroidal proximity to the interferometer. The sudden changes in density observed for LFS injection (green) at $3.003 \mathrm{~s}$ and $3.005 \mathrm{~s}$ show examples of fringe jumps. Accounting for the fringe jumps, the increase in density measured for LFS and HFS injection is within 10\%. The decreasing value of density at $3.006 \mathrm{~s}$ observed with HFS injection may be due to measurement error but this cannot be confirmed and it does not impact the findings. The density trace during the CQ was measured to be almost identical between the two valves. This indicates that both have similar fueling efficiency with the same number of particles assimilated.

Previous studies reported the HFS valve has relatively higher fueling efficiency at low injection amounts [28]. As the injected quantity was increased, saturation was achieved and both valves produced similar plasma density increases. The measurements presented in this work find almost equal assimilation from both valves for the "small" injection quantity. Although termed "small" in this report, the injection is over $10 \times$ the plasma inventory $\left(\sim 8.4 \cdot 10^{20}\right)$. Fast interferometry measurements indicate a factor of 6 increase in density, indicating $\sim 4 \cdot 10^{21}$ particles of the $\sim 9 \cdot 10^{21}$ injected were assimilated with both valves. Furthermore, this study was conducted with different discharge parameters, such as lower plasma current, and different injection gas mixtures (90\% deuterium mixtures in this study compared to pure neon in Pautasso 2015 [28]). It is therefore assumed that comparable fueling efficiency has already been achieved for the 


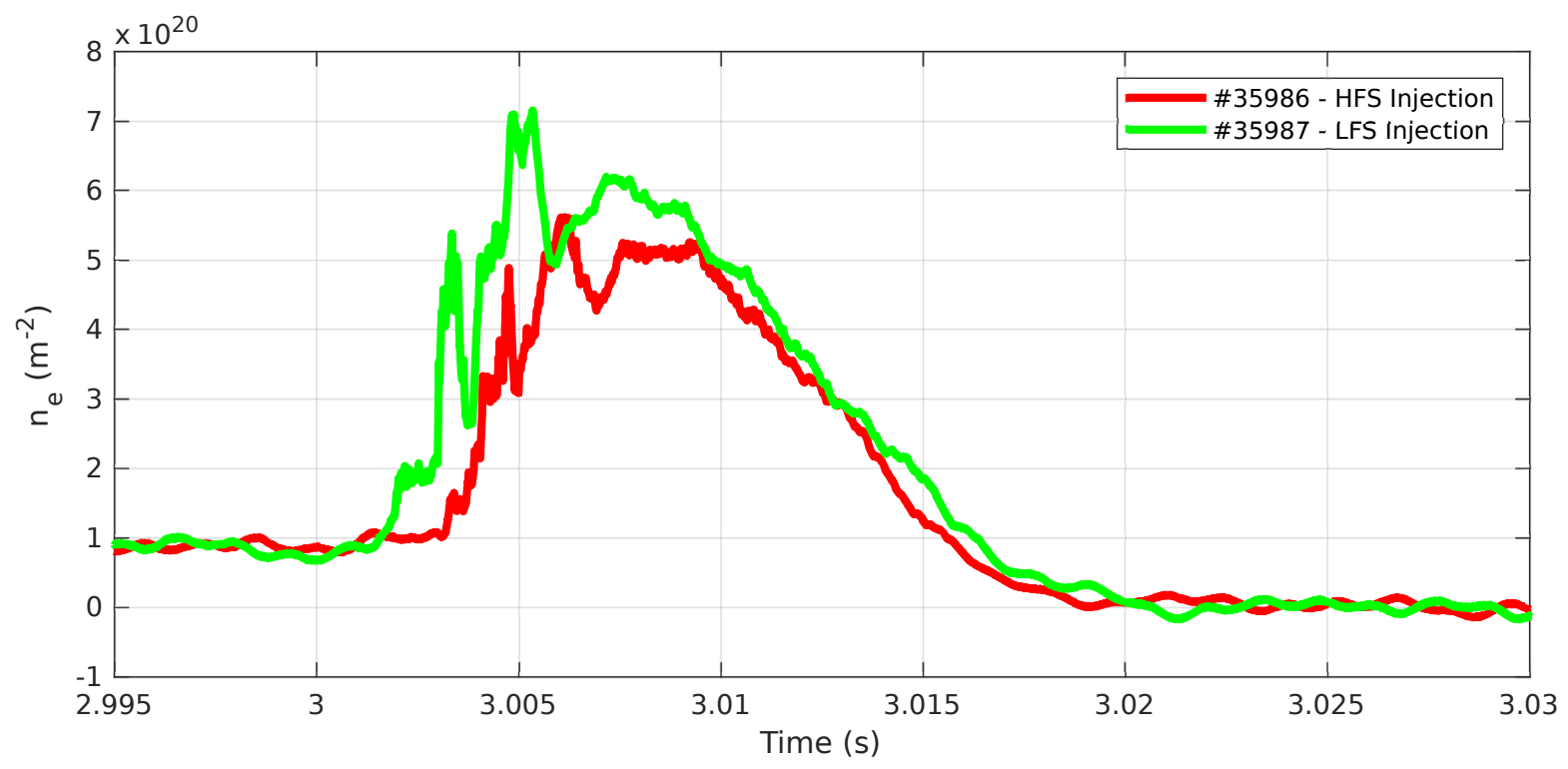

Figure 7. Density traces from the $\mathrm{CO} 2$ interferometer for "small" neon injections into high $W_{\text {th }}$ discharges with the HFS (red) and LFS (green) valves. The HFS injection has been shifted in time to match.

lower injection quantity that will remain comparable for the higher injection quantities.

The CQ time $\left(\tau_{\mathrm{CQ}}\right)$, linearly extrapolated from the $80 \%-20 \% \mathrm{CQ}$ time, can be used to approximate the relative assimilation of impurities into the plasma. This is possible as $\tau_{\mathrm{CQ}}$ is a function of plasma resistivity and self-inductance, assuming the plasma shape does not change significantly and there are no large vertical displacements [28]. Thus, a similar $\tau_{\mathrm{CQ}}$ for a given impurity would indicate a similar assimilated amount. Figure 8 presents $\tau_{\mathrm{CQ}}$ as a function of the average atomic number of the gas mixture (Mix. Z) and the number of injected particles. Both valves produced similar $\tau_{\mathrm{CQ}}$ for a given number of injected particles and Mix. Z, indicating the same assimilation efficiency for both valves for each gas. Saturation of $\tau_{\mathrm{CQ}}$ is not achieved for the smaller injection amount and as expected, a shorter $\tau_{\mathrm{CQ}}$ is measured for argon. Doubling the quantity of argon injected produced only a $\sim 15 \%$ decrease in $\tau_{\mathrm{CQ}}$. Doubling the quantity of injected neon reduced $\tau_{\mathrm{CQ}}$ by $\sim 75 \%$ in line with values comparable with large argon injection indicating saturation of assimilated particles has been achieved.

\subsection{Radiated Energy}

The AXUV diodes and bolometers were used to investigate radiation emission location, toroidal asymmetries and total radiated energy. The high temporal resolution of the AXUV diodes permits qualitative measurements of radiation emission location, impurity transport (poloidal and toroidal) and penetration depth. Figure 9 presents the temporal evolution of incident radiated power (on a logarithmic scale) per channel (diode) for the reference discharge. The three cameras presented are the DVC13 fan in sector 13 and the upper and lower LFS cameras in sector 5. 


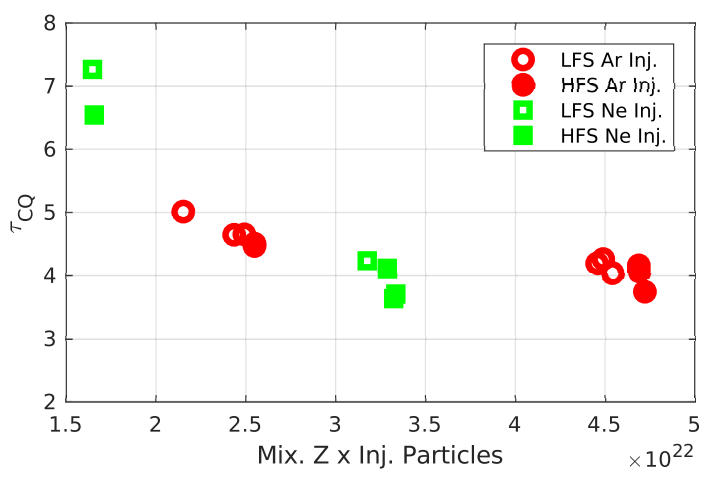

Figure 8. CQ time as a function of average atomic number of the gas mixture and the injected particles.
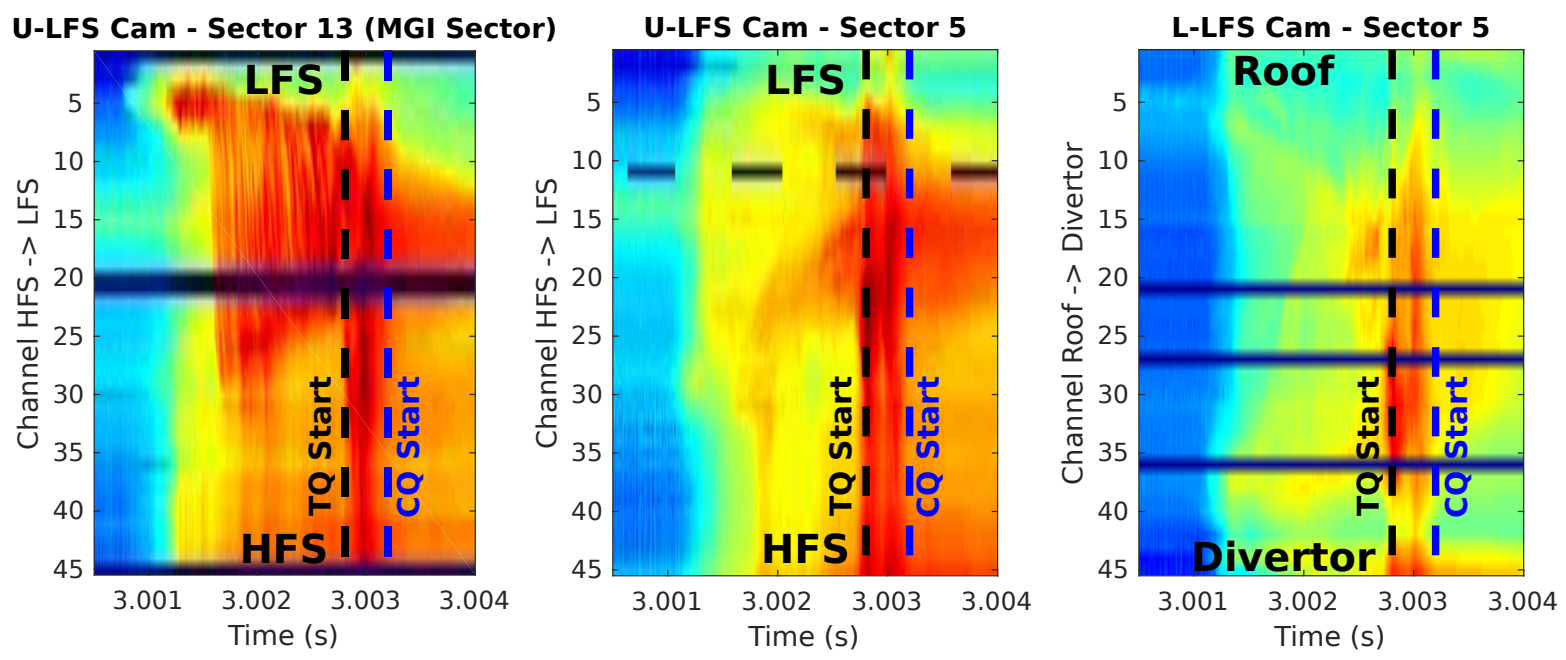

Figure 9. Temporal evolution of radiated power (on a logarithmic scale) per channel for the reference discharge (\#35653). Left - DVC13 fan in sector 13, middle - the upper LFS cameras in sector 5 , right - lower LFS camera in sector 5.

The emission from gas arrival at the LFS plasma edge is apparent from LFS injection on the AXUV camera in sector 13. The radiation is initially localised on the LFS and then produces a circulating pattern between the LFS and mid-plane until the TQ. This pattern is due to parallel impurity transport along helical field lines. An increase in radiation is measured in the opposite sector within $0.1 \mathrm{~ms}$ of gas arrival due to fast parallel transport of impurities. The relatively lower emission measured in the opposite sector is attributed to changes in the emission spectrum resulting in a relatively weaker response from the AXUV diodes that display strong spectral sensitivity behaviour.

The temporal evolution of radiated power for the three bolometer inference techniques and the AXUV diodes from both sectors are presented in Figure 10 for the reference discharge with LFS injection (\#35653) and a comparable discharge with HFS injection (\#35982). The AXUV diode signals have been scaled by a factor of 25 to match the radiated power from the bolometers. A scaling factor of $15 \mathrm{in} W_{\text {rad }}$ was observed 
during flat-top operation, so a factor of 25 during a disruption is not implausible [35]. The difference in temporal resolution between the two diagnostics is apparent from the sharp changes in radiated power inferred from the diodes. For LFS injection, the diodes in sector 13 measured an early increase in radiated power in the injection sector (13) and larger total radiated energy across the disruption relative to the diode measurements in sector 5. This is attributed to a relatively high spectral response of the diodes to visible photons (see Figure 4) and an analysis of toroidal asymmetries is presented in the following subsection. Measurements in sector 13 were not available during HFS injection discharges.
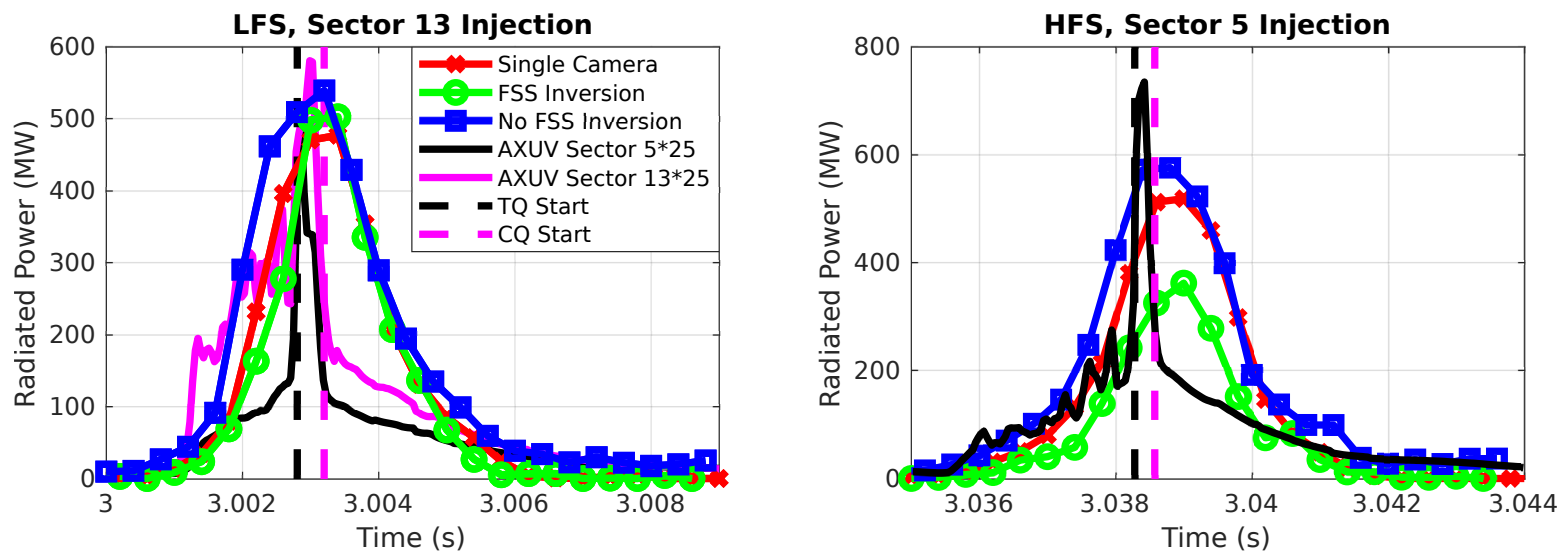

Figure 10. Radiated power from the three bolometer inference techniques and scaled AXUV diodes for LFS (left) and HFS (right) injection into comparable discharges (\#35653 and \#35982 respectively).

The bolometers measured a slower increase in radiated power over $2 \mathrm{~ms}$ by all three techniques with the inferred peak radiated powers are within $10 \%$ of each other for LFS injection. The no FSS inversion predicts the highest radiation in the pre-TQ phase and this was observed in all discharges. The source of this difference is attributed to variations in location of strong emission regions. The emission location during the CQ is expected to be more homogeneous and this is reflected by the comparable powers inferred after the TQ for LFS injection. The post TQ variation in radiated power for HFS injection is attributed to continued MGI injection from unempty reservoirs [28]. This results in localised radiation at the HFS and differences in inferred radiated power depending on the emission location calculated. The variation in inferred radiated power for localised and homogeneous plasma emission profiles illustrates the potential errors that can arise when using inversion codes benchmarked for standard operation during disruptions.

The sector 5 HFS injections are more challenging for the inversion algorithms as the radiation measurement is in the same sector, leading to strongly localised emission. The inverted profiles at peak power for the reference LFS and HFS injection discharges are presented in Figure 11. All four inversions place the bulk of radiation close to the HFS mid-plane and indicate significant radiation at the outer divertor. This implies that the 
impurities have not penetrated to the core at this time but have been transported along flux surfaces from the injection location. The streaks of high or zero emission across the inverted image with the no FSS technique are inversion artefacts and suggest weaker regularisation.
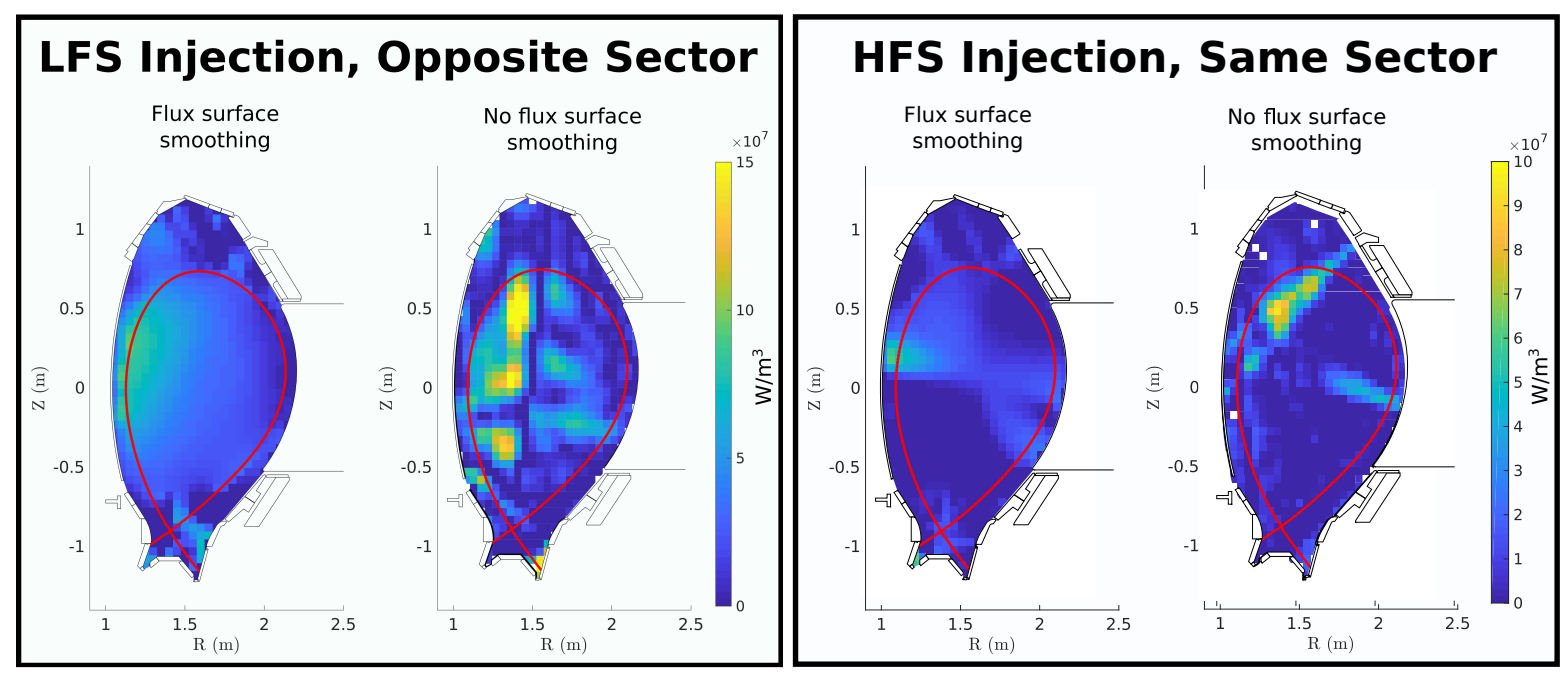

Figure 11. Tomographic inversions of bolometer data at peak radiation using the FSS method and the no FSS method for LFS (left) and HFS (right) injection into comparable discharges (\#35653 and \#35982 respectively).

The shapes of high radiating regions provide an insight into the influence of numerical smoothing along flux surfaces or R-z co-ordinates. An example of this is the smoothing along the HFS separatrix in the FSS inversion compared to strongly radiating regions in an almost vertical line across flux surfaces with the no FSS inversion for LFS injection. Stronger smoothing for the FSS inversion is able to better mask inversion artefacts from channels measuring increased localised radiation, which appear as streaks in the no FSS inversion. The disadvantage of strong smoothing is the reduction in spatial precision and radiation pattern localisation such as that observed in the outer divertor with the no FSS inversion.

The consistently higher radiated power computed with the no FSS inversion is attributed to the emission location being placed further inside the plasma at a larger radius. Consequently, a larger radiating volume is calculated and leads to a higher radiated power. Similarly, the single camera technique is expected to overestimate the radiated power as it assumes constant radiation emission along a flux surface, which leads to the localised radiation at the HFS being averaged across the entire flux surface. The impact of these factors on uncertainty in radiated power is discussed in Section 3.2 .2 .

Figure 12 presents the total $W_{\text {rad }}$ integrated between the arrival of gas and the end of the CQ. The ratio between the inversion techniques varied from 1.2 to 2 in the LFS MGI mitigated discharges, with the single camera inferred $W_{\text {rad }}$ fluctuating between the values from the inversions. The no FSS technique consistently predicted the highest 
$W_{\text {rad }}$, whereas the FSS inversion consistently predicts the lowest. This result highlights the large variation possible in $W_{\text {rad }}$ based on the inversion method applied and the need for further development of inversion methods.
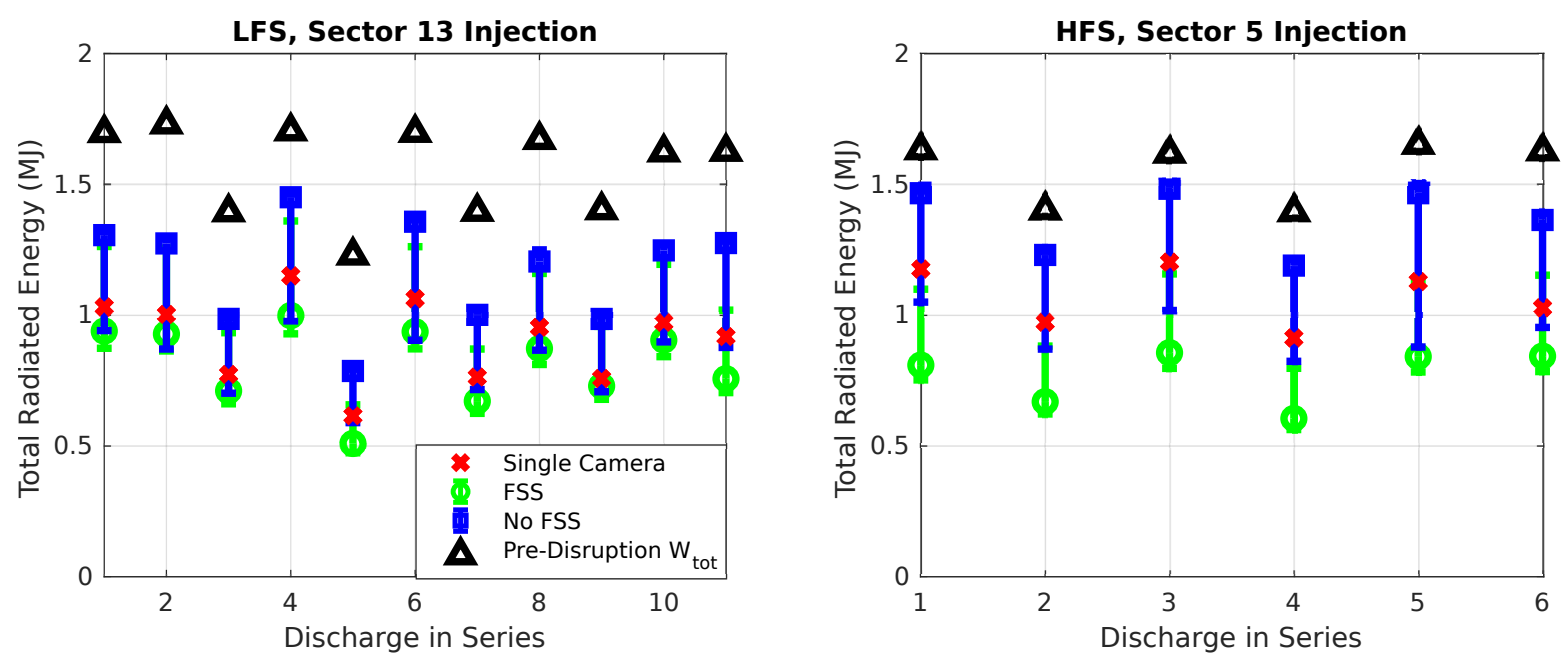

Figure 12. Total radiated energy for LFS MGI discharges (left) and HFS MGI discharges for the three bolometer inference techniques.

The ratio between the calculated radiated energies is approximately constant when using the HFS valve, which is in the same sector as the bolometers. The consistently higher $W_{\text {rad }}$ from the no FSS technique is attributed to localisation of emission at larger radius and poorer convergence of the inversion. This is also demonstrated by the error bars (detailed in Section 3.2.2). The absolute values of $W_{\text {rad }}$ with the no FSS inversion are over $90 \%$ of the total pre-disruption energy $\left(W_{\text {th }}+W_{\text {mag }}\right)$, implying less than $10 \%$ of the total energy was deposited on the divertor and coupled to the structure during the CQ. This is unrealistically low based on the previously estimated $30 \%$ of $W_{\text {mag }}$ coupling to the vessel structure and coils on AUG, 20-60\% on JET and 50\% on DIII-D [28, 36]. Based on the unrealistically high $W_{\text {rad }}$ inferred, the no FSS inversion will not be used in the energy balance.

\subsubsection{Radiation Asymmetries}

The assumption of toroidal symmetry cannot always be applied in mitigated plasmas due to injection at a single toroidal location. The level of toroidal asymmetry in these discharges was investigated using MGI valves in opposite sectors of the machine. The assimilated fraction was shown to be equal for both valves in Section 3.1 and thus the $W_{\text {rad }}$ is expected to be approximately equal between the valves for a given gas type and quantity. It therefore follows that any difference measured in $W_{\text {rad }}$ for the same injection is a measure of toroidal radiation asymmetry.

Figure 13 left presents $W_{\text {rad }}$ inferred from the FSS analysis assuming no toroidal asymmetries. With the exception of one outlier at 1.05 MJ, comparable radiated energies are measured between the LFS (empty symbols) and HFS (filled symbols) injections for 
a given Mix. Z and injection quantity. This implies the same $W_{\text {rad }}$ was measured in the injection and toroidally opposite sectors and thus toroidal asymmetries are negligible in the mitigated discharges presented. Similar results have been reported on DIII-D with toroidal peaking factors of $1.2 \pm 0.1$ during the TQ phase, implying a lower toroidal peaking factor over the entire disruption sequence [37].
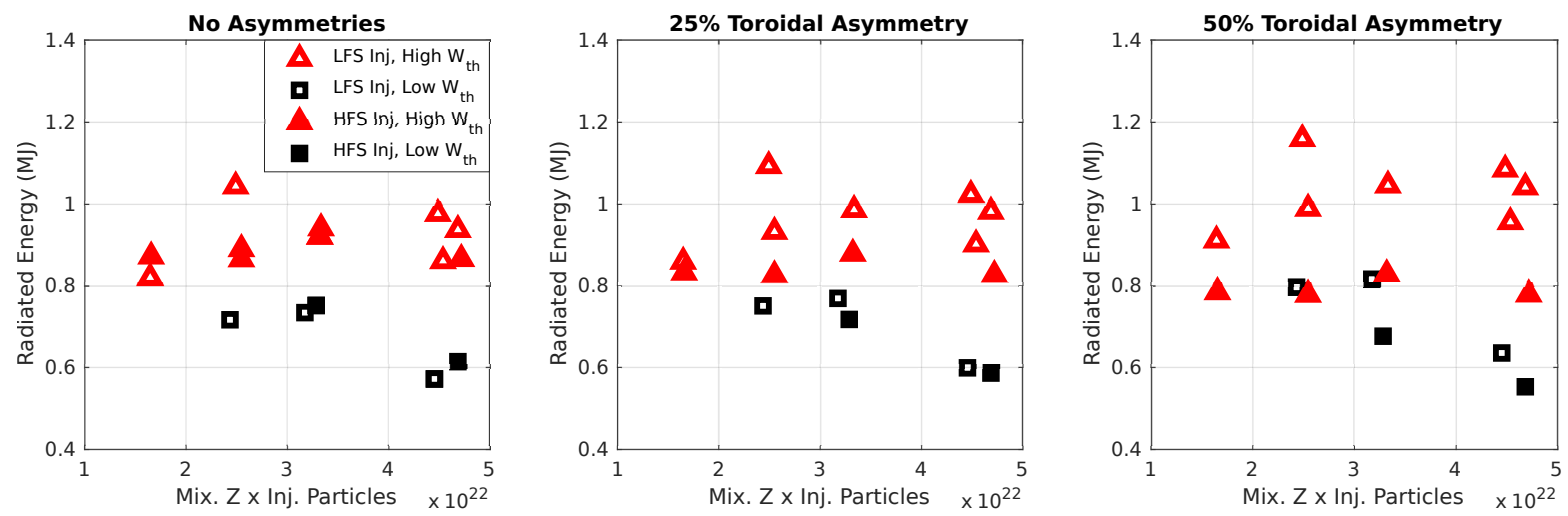

Figure 13. Radiated energy as a function of injected gas composition and quantity assuming $0 \%, 25 \%$ and $50 \%$ toroidal asymmetries from left to right.

The sensitivity of this methodology was investigated by artificially imposing $25 \%$ (Figure 13 middle) and 50\% (Figure 13 right) toroidal asymmetries. A simple linear peaking profile was applied to calculate the $W_{\text {rad }}$ presented. The calculated $W_{\text {rad }}$ for the same injection (gas type and quantity) diverges as the asymmetry factor is increased and this could only occur if the same assimilated gas led to significantly different values of $W_{\text {rad. }}$. Imposing a Gaussian peaking profile for the same analysis leads to stronger peaking in the injection sector and thus further divergence in $W_{\text {rad }}$ between the two valves for the same injection. This sensitivity study indicates that a $50 \%$ toroidal asymmetry would produce a $\sim 25 \%$ difference in $W_{\text {rad }}$ for the same assimilated gas type and quantity. This result is implausible and supporting the finding that toroidal asymmetries are negligible in the mitigated discharges presented.

\subsubsection{Uncertainties in Radiated Energy}

Highly localised radiation emission and fast transient events can lead to significant uncertainties in $W_{\text {rad }}$. The following section presents an analysis aimed at quantifying uncertainties in the techniques used to infer $W_{\text {rad }}$ and selecting the most appropriate one for the energy balance. Errors in the alignment of the cameras, capture angle, calibration and sensitivity variation between foils are assumed to be negligible relative to the errors in the inference techniques. The uncertainties for the AXUV diodes will not be discussed here as they are relatively calibrated using the bolometers and the impact of their photon energy dependent response is discussed in the relevant sections.

The single camera inference technique assumes constant emission along flux surfaces and this assumption is not appropriate for the highly localised radiation patterns in mitigated disruptions. Previous analyses using phantom reconstructions of standard 
plasma emission profiles found a systematic underestimation of radiated power by up to $39 \%$ [33]. As previously discussed, localised radiation at the HFS leads to a significant overestimation during mitigated disruptions. Thus the uncertainties for the single camera technique are assumed to be significantly higher than those of the FSS and no FSS inversion methods and will not be further analysed here.

Three significant contributors to the uncertainty of total radiated power for the inversion techniques are the convergence of the inversion, radial position of high emission regions and the position or presence of negative emission regions. A direct comparison to determine the accuracy of a tomographic inversion is the reconstruction of radiation along each line of sight. The variation with respect to measured values can be used to estimate the quality of the match and convergence of the inversion. The reconstruction error is separated into positive and negative errors at each time point and the positive and negative integrals are presented separately in Figure 14. A positive error indicates $W_{\text {rad }}$ is under-predicted and a negative error indicates overestimation. This analysis suggests that $W_{\text {rad }}$ is systematically overestimated by the no FSS inversions and underestimated by the FSS inversions. The no FSS inversion overestimates by up to $21 \%$ compared to up to $17 \%$ under-prediction by the FSS inversions. There are certain time points where the no FSS technique underestimates the radiation and this is indicated by the positive errors in the integrals. There are no time points at which the FSS analysis provided a negative error.
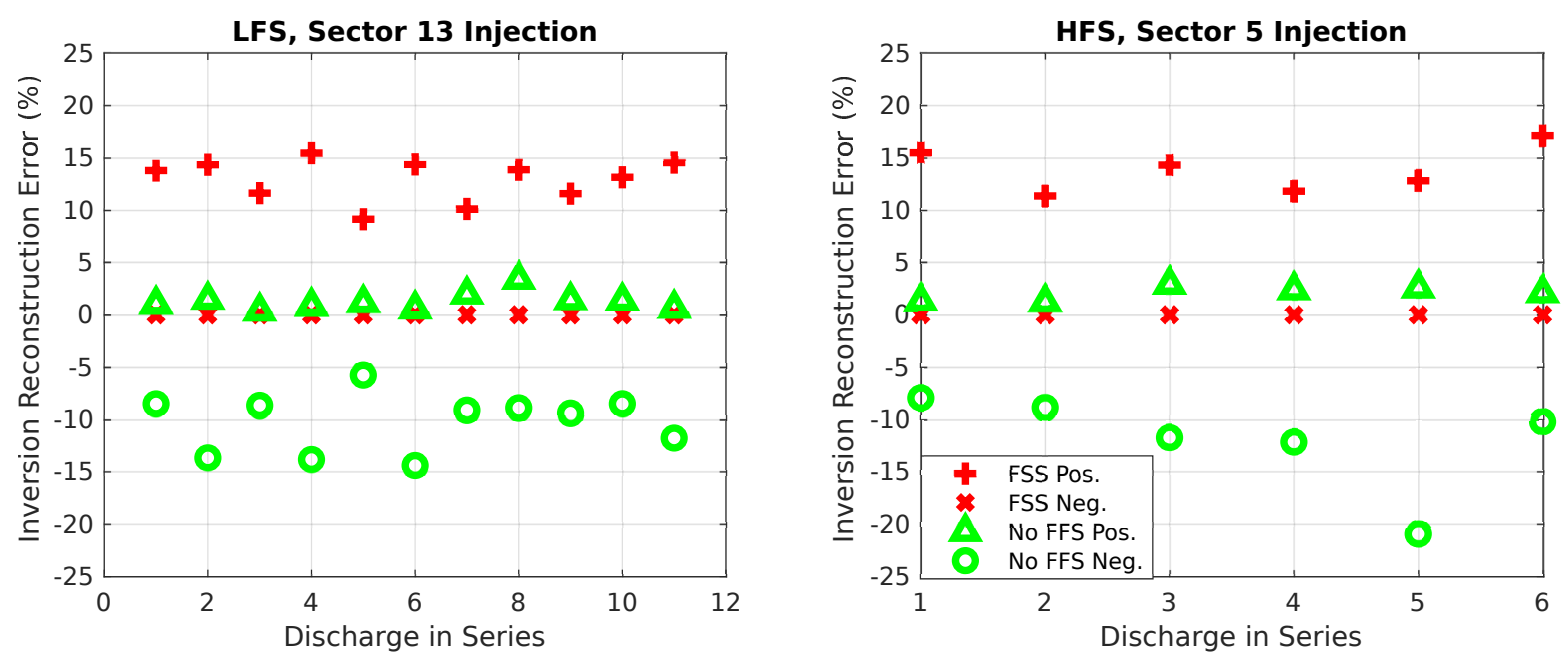

Figure 14. Error in reconstructed line of sight radiation for the bolometer inversion techniques. A positive error indicates under-prediction of $W_{\text {rad }}$.

The reconstructed location of strong emission zones can have a significant impact on the inference of $W_{\mathrm{rad}}$. The most sensitive factor is the radial position as this can alter the volume of the radiating plasma. An example of this is shown in Figure 11 where the FSS method reconstructs a strong emission region close to the HFS wall and the no FSS method places it further inside the plasma. The difference in radial location from $\sim 1.20 \mathrm{~m}$ to $\sim 1.45 \mathrm{~m}$ results in a variation of radiating volume of $\sim 20 \%$. This trend is 
Table 2. Uncertainties in $W_{\text {rad }}$ inferred from bolometric inversions.

\begin{tabular}{|l|c|c|}
\hline Uncertainty Source & FSS & No FSS \\
\hline Inversion convergence (maximum) & $+17 / 0 \%$ & $+4 /-21 \%$ \\
\hline Localisation of high radiation zones & $+20 /-0 \%$ & $+0 /-20 \%$ \\
\hline Location of negative values & $+0 /-7 \%$ & N/A \\
\hline Total & $+37 /-7 \%$ & $+4 /-41 \%$ \\
\hline
\end{tabular}

repeated in all inversions studied and partially accounts for higher $W_{\text {rad }}$ inferred with the no FSS technique. The uncertainty from radial location is therefore estimated to be $+20 \%$ for the FSS method and $-20 \%$ for the no FSS method.

The FSS inversion technique permits negative emission regions to reduce computation time. They are included in the calculation of total radiated power but this been shown to produce no significant errors during standard operation [31]. However, in mitigated plasmas with highly poloidally asymmetric emission profiles, the location of negative emission regions can contribute to errors in the total radiated power. The absolute values of negative emission were found to be typically one third of the positive emission when the radiated power was above $50 \mathrm{MW}$ and the location was generally observed to be at the LFS. Consequently, a total $W_{\text {rad }}$ uncertainty of $-7 \%$ was estimated from the radial emission location analysis.

A summary of the uncertainties produced by the factors discussed are presented in Table 2. The individually calculated errors from the inversion reconstruction were coupled with the estimated uncertainties due to emission location to provide the values presented in Figure 12. Most of the uncertainty range is overlapped by both inversion techniques for LFS injection indicating the possibility of using the two techniques together to better constrain $W_{\text {rad }}$. The larger discrepancy when using the HFS valve indicates the no FSS method is not well suited to localised emission at the HFS and this is assumed to be the reason for the unrealistically high total radiated energies computed (over $90 \%$ of the total pre-disruption energy). Therefore, the FSS inversion will be used for the remaining analysis presented in this study whilst noting that these inferences are likely under predicting the total $W_{\text {rad }}$.

\section{Energy Balance}

The energy balance methodology applied in this study is based on the model presented in Lehnen 2013 [23]. The two unknowns in the disruption energy balance are the total $W_{\text {rad }}$ and $W_{\text {coupled }}$. Section 3.2 outlined the process used to constrain $W_{\text {rad }}$ with the bolometers leaving $W_{\text {coupled }}$ as the remaining unknown. This section presents a novel method to estimate $W_{\text {coupled }}$ from diagnostic measurements on AUG and provide a comparison for simulations of these discharges currently being carried out to calculate $W_{\text {coupled }}$.

The high temporal resolution AXUV diodes were utilised to estimate the fraction of total radiation emitted prior to the start of the $\mathrm{CQ}$, determined by the spike in plasma 
current. These fractions were multiplied by the $W_{\text {rad }}$ measured over the entire disruption to estimate $W_{\text {rad }}$ prior to and during the CQ. The AXUV array in the toroidally opposite sector was used instead of the array in the injection sector to minimise the influence of the high relative sensitivity to visible photons (see Figure 4). As the sector 13 AXUV system was not available during the session with HFS injection, this analysis could only be applied to the LFS injection discharges. The results of this analysis are presented as fractions of $W_{\text {th }}$ and $W_{\text {mag }}$ radiated in Figure 15.

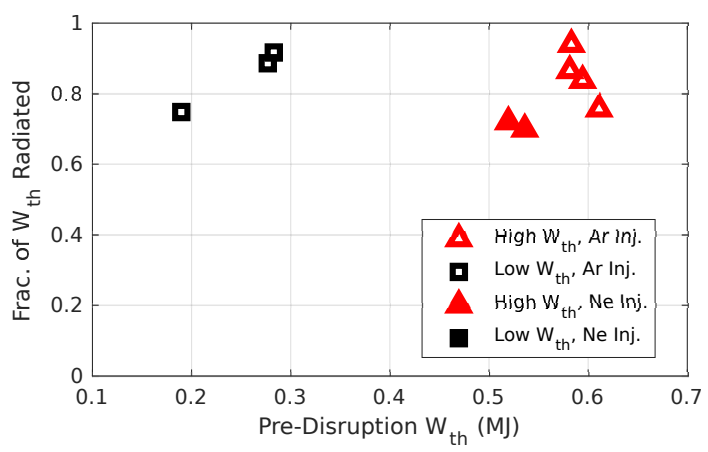

Figure 15. Fraction of $W_{\mathrm{th}}$ radiated as a function of pre-disruption $W_{\mathrm{th}}$.

Radiated $W_{\text {th }}$ fractions of 0.7-0.95 were inferred for the bulk of the discharges. Argon injections produced $\sim 10 \%$ higher radiated fractions at high pre-disruption $W_{\text {th }}$ that is likely due to the higher radiation efficiency of argon and/or the relative spectral response of the diodes for the two gases. No decrease in fraction of radiated $W_{\text {th }}$ was observed with increasing pre-disruption $W_{\text {th }}$.

The fraction of $W_{\text {th }}$ that is not radiated is expected to be deposited on the divertor during the TQ. A comparison between $W_{\text {th }}$ deposited and all three IR cameras was not possible as injected impurities resulted in significant radiation being recorded that masked the real target temperature. The camera observing two optimised tiles on the outer divertor was able to produce usable images at $1.5 \mathrm{~ms}$ temporal resolution. Figure 16 shows three sequential frames from this camera showing the strike point at gas arrival (top), beginning of the TQ (mid) and during the CQ at approximately $600 \mathrm{kA}$ (bottom) for the reference discharge \#35653. The strike point on both tiles can be clearly located by the localised higher temperature. The increase in baseline temperature in the second image is ascribed to plasma radiation and their reflections.

Figure 17 presents the maximum change in average tile surface temperature (dT) for a given $W_{\text {th }}$ during the disruption. It should be noted that the relatively low temporal resolution can result in the $\mathrm{dT}$ being measured up to $1.5 \mathrm{~ms}$ after the end of the TQ, which can lead to an underestimation of TQ dT. An increase in dT was observed with increasing $W_{\text {th }}$ deposited for argon injections and the neon injection disruptions produced a relatively lower dT for their estimated deposited $W_{\text {th }}$.

This is attributed to the variation in spectral mission of the two gases coupled to strong AXUV spectral sensitivity, influencing the radiated $W_{\text {th }}$ estimate. The extrapolated dT with no deposited energy is $100 \mathrm{~K}$ and this offset is attributed to 

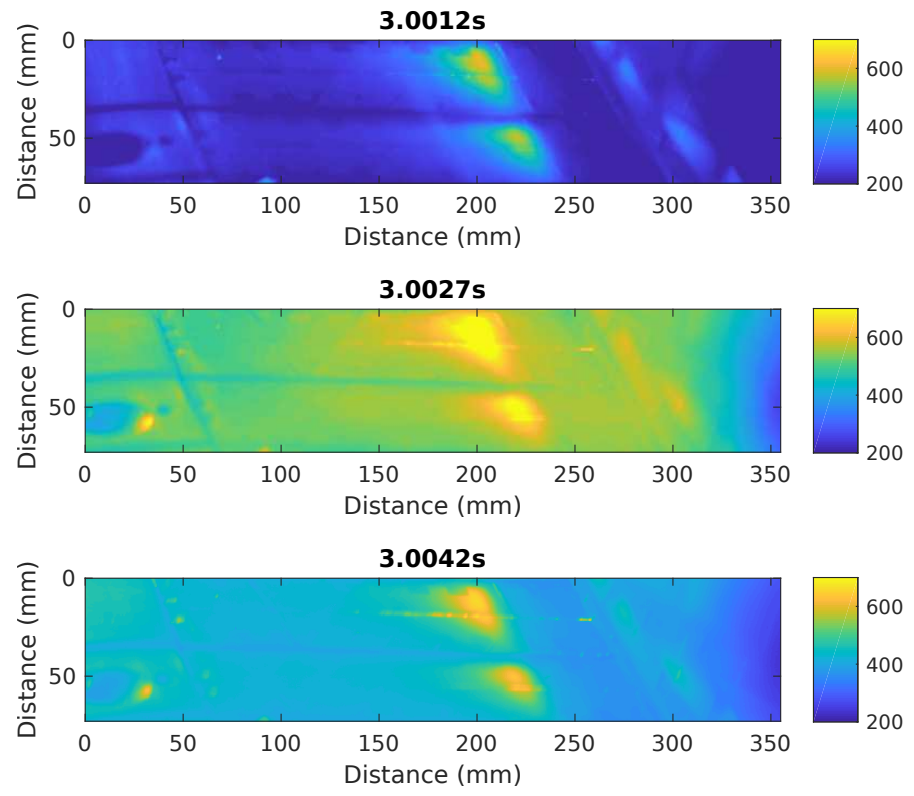

Figure 16. Left - IR images of the outer divertor target tiles during the reference discharge (\#35653) at gas arrival (top), beginning of the TQ (mid) and during the CQ at approximately $600 \mathrm{kA}$ (bottom).

a combination of background plasma emission and reflections being recorded on the camera.

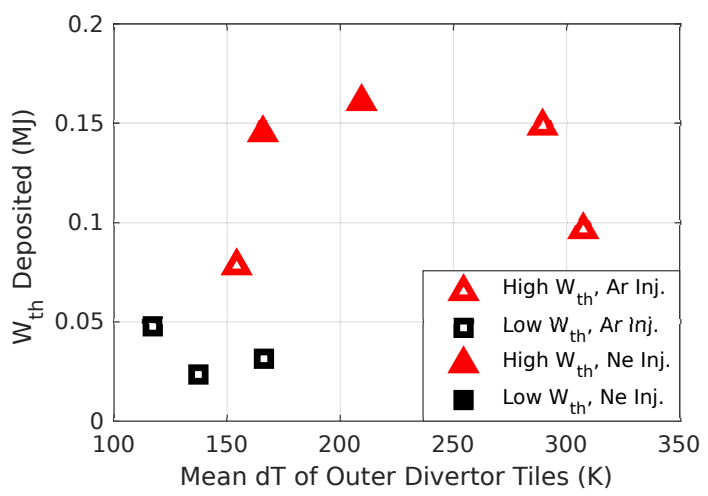

Figure 17. Maximum change of average outer divertor target temperature during the disruption as a function of deposited $W_{\mathrm{th}}$.

The fraction of radiated $W_{\text {mag }}$ is presented in Figure 18 and was approximately constant at 0.4 for all CQ times with no difference in radiated $W_{\text {mag }}$ fraction observed between the two injection gases. The balance between radiated, deposited and coupled $W_{\text {mag }}$ was explored in Lehnen [23]. It was shown that MGI mitigated plasmas without a vertical displacement event resulted in short CQ times normalised to the plasma crosssection area $\left(2-10 \mathrm{~ms} / \mathrm{m}^{2}\right)$. The time scales required for Ohmic heating of the plasma and heat conduction to the wall during the CQ were of the order of $\left(100 \mathrm{~ms} / \mathrm{m}^{2}\right)$ and only occurred in unmitigated disruptions with the ITER like wall. The plasma surface area was $\sim 1.1 \mathrm{~m}^{2}$ for all the discharges presented and the normalized CQ times are 
3.2-6.4 ms $/ \mathrm{m}^{2}$. These short normalized CQ times imply they are strongly radiation dominated and any $W_{\text {mag }}$ not radiated can be assumed to be coupled to the vessel structure and coils.

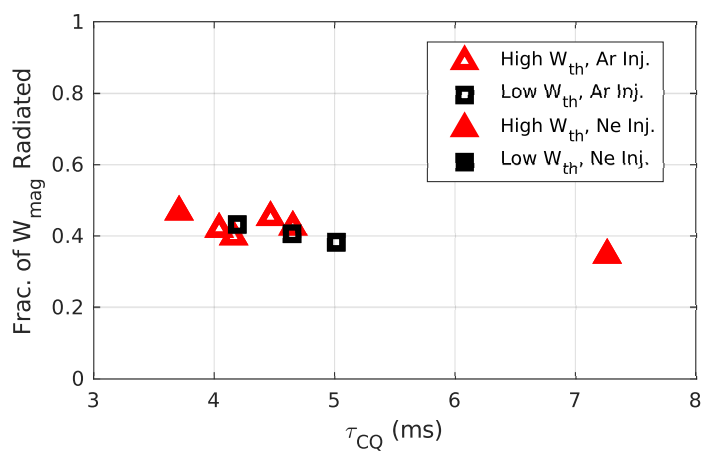

Figure 18. Fraction of $W_{\mathrm{mag}}$ radiated as a function $\tau_{\mathrm{CQ}}$.

The discharges produced an almost constant radiated $W_{\text {mag }}$ fraction of $\sim 0.4$ and thus a $W_{\text {coupled }}$ fraction of $\sim 0.6$. This is comparable with an almost constant $W_{\text {coupled }}$ fraction of 0.5 reported across a range of discharges on DIII-D and over 0.6 on JET $[36,23]$. Halo current $\left(I_{\text {halo }}\right)$ measurements from the inner divertor shunt resistor and eight strain gauges mounted on the vertical tokamak support columns were used to validate the constant inferred $W_{\text {coupled. }}$. Figure 19 presents times traces for both of these diagnostics. The sum of all eight strain gauges provided an estimate of the net vertical force the ignored distortion and twisting forces. The signal was zeroed at the time just before the disruption to measure only the vertical forces caused by the disruption by compensating for the strain measured from the load of the vessel itself.

Increases in $I_{\text {halo }}$ were observed during the $\mathrm{CQ}$ and the peak value was comparable for most discharges. The $I_{\text {halo }}$ profile for the final discharge in the LFS injection dataset, indicated by the dashed magenta line, is relatively offset in time that is attributed to the longer CQ time. Similarly, the last two discharges in the HFS injection dataset are also relatively offset in time. These two discharges produced relatively higher peak $I_{\text {halo }}$ that is attributed to a vertical movement of the plasma. Consequently, the highest vertical forces were measured for these two discharges.

\subsection{Radiated Energy Fraction}

The $W_{\text {rad }}$ from FSS inversions with inferred $W_{\text {coupled }}$ from the AXUV diodes was used to calculate $f_{\text {rad }}$ and $f_{\text {th }}$ as described in Equations 1 and 2. A value of 0.60 was used for the $W_{\text {coupled }}$ fraction for discharges where the AXUV diodes failed to provide a result. The calculated values of $f_{\text {rad }}$ are presented in Figure 20, with the empty red circle markers indicating LFS injection and filled red circle markers for HFS injection. Values of $f_{\mathrm{rad}}$ ranging from 0.8 to 1.0 were calculated with no reduction in $f_{\text {rad }}$ as $f_{\text {th }}$ increased. The error bars presented represent solely the radiated energy uncertainty and do no include uncertainties in $W_{\text {coupled }}$. 

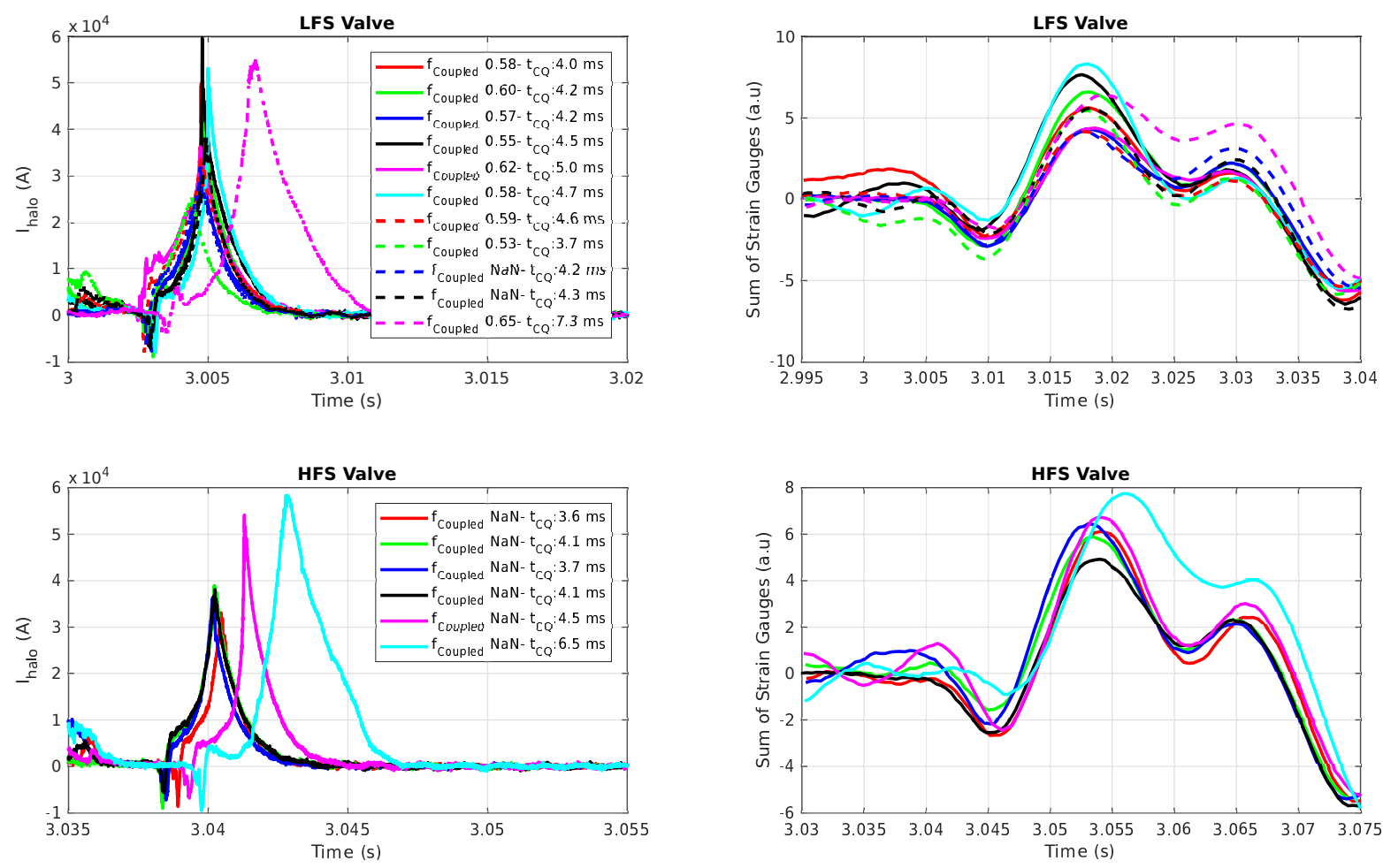

Figure 19. Left - $I_{\text {halo }}$ measured at the inner divertor shunt for all discharges. Right - Sum of all eight strain gauges mounted on the vertical vessel support columns.

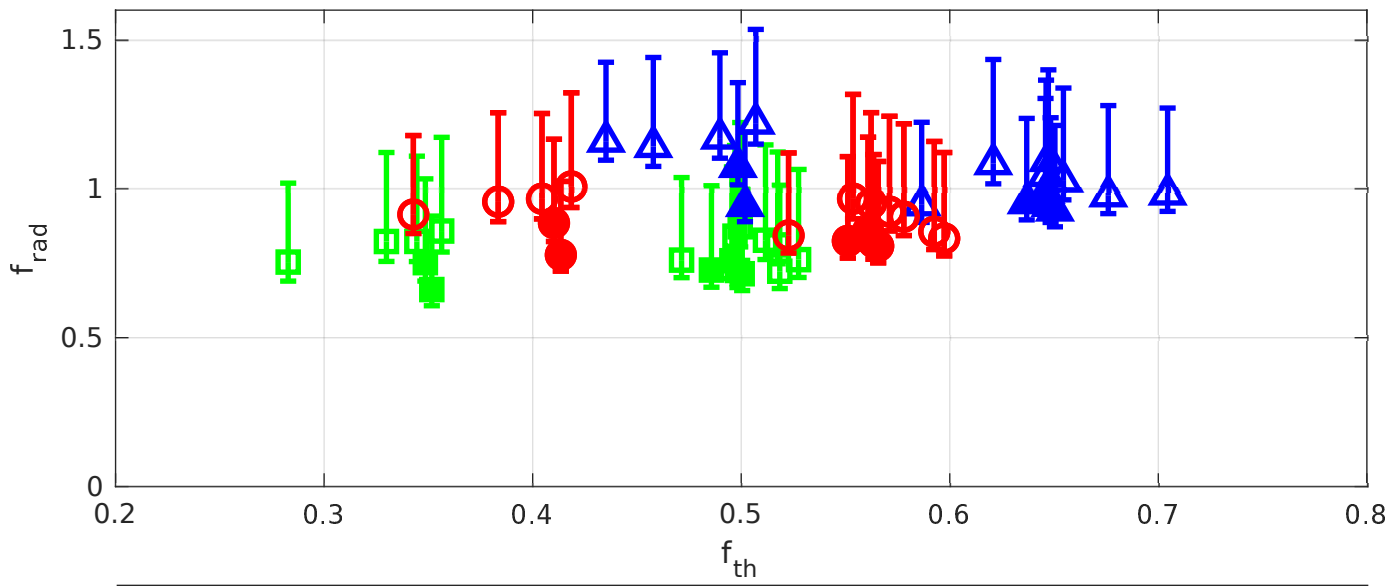

LFS Inj. Inferred $W_{\text {coupled }}$
LFS Inj. $20 \%$ Reduction of $W_{\text {coupled }}$
$4 \quad$ LFS Inj. $20 \%$ Increase of $W_{\text {coupled }}$

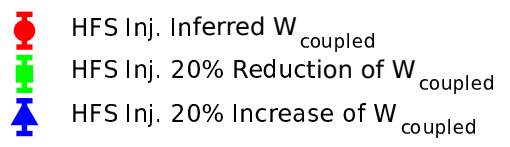

Figure 20. Computed $f_{\text {rad }}$ as a function of $f_{\text {th }}$ for LFS (empty markers) and HFS (filled markers) injection. Red circle markers - $f_{\text {rad }}$ computed with inferred $W_{\text {coupled }}$ from the AXUV diodes. Blue triangle and green square markers - $f_{\text {rad }}$ computed with an artificial $20 \%$ increase and decrease in $W_{\text {coupled }}$ respectively. 
The photon energy dependent sensitivity of the AXUV diodes leads to uncertainties in the estimation of $W_{\text {rad }}$ prior to and during the CQ. For example, it is shown in Figure 4 that the diodes have higher sensitivity to visible photons than UV photons. If more visible light is emitted prior to the CQ, this would alter the ratio and lead to an underestimation of CQ $W_{\text {rad }}$ and an overestimation of $W_{\text {coupled }}$. It is difficult to constrain this uncertainty without fast spectrally resolved measurements but the impact of such an error can be explored through the variation of $W_{\text {mag }}$ radiated or coupled. A $20 \%$ overestimation of radiated $W_{\text {mag }}$, due to relatively higher sensitivity of the diodes during the CQ, would result in a $20 \%$ underestimation of $W_{\text {coupled }}$. The impact a $\pm 20 \%$ error in $W_{\text {coupled }}$ on $f_{\text {rad }}$ is presented with the blue triangle and green square markers in Figure 20.

An underestimation of $W_{\text {rad }}$ during the CQ would lead to an overestimation of

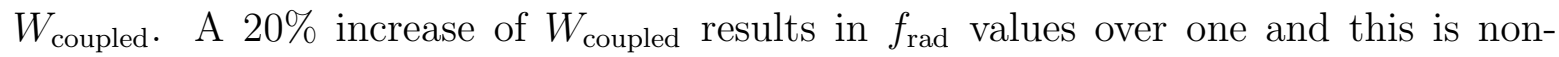
physical (over $100 \%$ of $W_{\text {th }}$ radiated). This clearly indicates that $W_{\text {rad }}$ during the CQ is not being significantly underestimated. A $20 \%$ decrease of $W_{\text {coupled }}$, due to a potential $20 \%$ overestimation of $W_{\text {rad }}$ during the CQ, leads to a decrease in $f_{\text {rad }}$ but does not alter the constant $f_{\text {rad }}$ found for a range of $f_{\text {th }}$ values.

An additional constraint of the radiation dominated $\mathrm{CQ}$ model applied is the convergence of $f_{\text {rad }}$ to one as $f_{\text {th }}$ approaches zero[23]. As $f_{\text {th }}$ approaches zero, the total plasma energy is effectively $W_{\text {mag }}$, which can only be radiated or coupled and thus $f_{\text {rad }}$ must equal one. The almost constant $f_{\text {rad }}$ value of one with varying $f_{\text {th }}$ implies these discharges are well mitigated and the trend meets the constraint discussed. The size of the error bars on the radiated energy measurement do not allow for further convergence on $W_{\text {coupled }}$, however, it can be concluded that the $W_{\text {coupled }}$ is likely between $48-60 \%$ of $W_{\text {mag }}$. Within the range of $W_{\text {coupled }}$ explored, no variation of $f_{\text {rad }}$ as a function of $f_{\text {th }}$ was found.

\section{Conclusions}

This study has produced a disruption mitigation dataset targeting a constant plasma scenario with varying auxiliary power. Scans of two injection quantities with $90 \%$ deuterium and 10\% neon or argon gas mixtures were performed at two nominal values of $f_{\text {th }}$ and with two MGI valves at different toroidal and poloidal locations. This study investigated MGI assimilation, influence of bolometer inversion techniques of fast evolving radiation patterns, estimates of $W_{\text {coupled, }}$ radiation asymmetries and the disruption power balance that can be achieved on AUG with current diagnostics.

Three techniques to infer $W_{\text {rad }}$ using bolometers showed variations of up to a factor of two. These differences were attributed to convergence limitations and radial positions of strong emission locations. This highlight the large uncertainty in $W_{\text {rad }}$ measurements with the current techniques and suggests this uncertainty could be reduced through emission location inferences from additional diagnostics. The FSS method was selected as the most reliable for emission profiles strongly peaked at the HFS and used to 
investigate toroidal asymmetries. The fueling efficiency was found to be equal for the two valves. The CQ times and $W_{\text {rad }}$ measured in the injection sector and toroidally opposite sector were independent of the valve used. Thus, asymmetries were estimated to be negligible for these discharges.

The AXUV diodes cross-calibrated with the bolometers were used to estimate the fractions of $W_{\text {th }}$ and $W_{\text {mag }}$ radiated. The difference between the pre-disruption $W_{\text {th }}$ and $W_{\text {rad }}$ before the end of the TQ was assumed to be deposited on the targets. The radiated thermal energy fraction was found to be $75-95 \%$ and the deposited energy correlated qualitatively with changes in tile surface temperatures. The inferred fraction of $W_{\text {mag }}$ coupled was $\sim 0.6$ and approximately constant compared to $\tau_{\mathrm{CQ}}$. This result was supported by the halo currents measured at the inner divertor and the vertical vessel forces.

The final energy balance produced $f_{\text {rad }}$ values between 0.8 and 1.0 with no reduction in $f_{\text {rad }}$ as $f_{\text {th }}$ increased. The impact of an error in the inferred $W_{\text {coupled }}$ was explored via artificial variation of $W_{\text {coupled }}$ by $\pm 20 \%$. An increase in $W_{\text {coupled }}$ produced non-physical results (over $100 \%$ of $W_{\text {th }}$ radiated before the CQ). A $20 \%$ decrease in $W_{\text {coupled }}$ produced plausible results and was estimated to be close to the lower limit of $W_{\text {coupled }}$ for these discharges. This analysis suggests $W_{\text {coupled }}$ for these discharges is likely between 48$60 \%$. Variations in $W_{\text {coupled }}$ within this range did not alter the almost constant $f_{\text {rad }}$ as a function of $f_{\mathrm{th}}$.

\section{Acknowledgements}

This work has been carried out within the framework of the EUROfusion Consortium and has received funding from the Euratom research and training programme 2014-2018 and 2019-2020 under grant agreement No 633053. The views and opinions expressed herein do not necessarily reflect those of the European Commission. This work was supported in part by the Swiss National Science Foundation. The authors would like to thank Alexander Bock for his help with the interferometry analysis.

\section{Bibliography}

[1] Lehnen, M., et al 2009 J. Nucl. Mater. 740 390-391

[2] Federici, G., et al 2001 J. Nucl. Mater. 290 260-265

[3] Putvinski, S., et al in Fusion Energy 2010 (Proc. 23rd Int. Conf. Daejeon, 2010) (Vienna: IAEA) CD-ROM file [ITR-1-6] and http://wwwnaweb.iaea.org/napc/physics/FEC/FEC2010/index.htm

[4] Lehnen, M., et al 2011 Nucl. Fusion $\mathbf{5 1 . 1 2} 123010$.

[5] Arnoux, G., et al 2011 J. Nucl. Mater. 415 S817

[6] Whyte, D.G., et al 2007 J. Nucl. Mater. 363-365 1160-7

[7] Pautasso, G., et al 2009 Plasma Phys. Control Fusion 51124056

[8] Hollmann, E.M., et al 2008 Nucl. Fusion 48115007

[9] Bakhtiari, M., et al 2005 Nucl. Fusion 45 318-25

[10] Thornton, A.J., et al 2011 J. Nucl. Mater. S836 415

[11] Luo, Y. H., et al 2014 Rev. Sci. Instruments 85.8: 083504 
[12] Dong, Y. B., et al 2014 No. NIFS-PROC-97

[13] Commaux, N., et al 2010 Nucl. Fusion 50112001

[14] Baylor, L.R., et al 2019, APS YP10-061

[15] Park, S.H., et al 2020 Fusion Eng. and Design 154111535

[16] Li, Y., et al 2018 Rev. Sci. Instruments 89.10: $10 \mathrm{~K} 116$

[17] Xu, H. B., et al 2019 Fusion Science and Technology 75.2:98-103

[18] Hollmann, E.M., et al 2015 Phys. Plasmas 22021802

[19] Commaux, N., et al 2016 Nucl. Fusion 56.4 046007

[20] Lehnen, M., et al 2015 J. Nucl. Mater. 39-48 463

[21] Jachmich, S., et al 2016 The 22nd PSI-conference

[22] Joffrin, E., et al "Disruption studies in the JET metallic wall". Preprint: 2016 IAEA Fusion Energy Conf, Kyoto [EX/9-1]

[23] Lehnen, M., et al 2013 Nucl. Fusion 53093007

[24] Bernert, M., et al 2014 Rev. of Sci. Instruments $\mathbf{5 3 . 3} 104003$

[25] Shiraki, D., et al 2018 APS DPPN11115S

[26] Neu, R., et al 2007 Plasma Phys. Control. Fusion 49 B59

[27] Meyer, H., 2019 Nucl. Fusion 59112014

[28] Pautasso, G., et al 2015 Nucl. Fusion 55.3033015

[29] Pautasso, G., et al in Fusion Energy 2008 (Proc. 22nd Int. Conf. Geneva, 2008) (Vienna: IAEA) CD-ROM file [EX/P9-1] and http://wwwnaweb.iaea.org/napc/physics/FEC/FEC2008/html/index.htm

[30] Meister, H., 2013 Rev. of Sci. Instruments 84123501

[31] Fuchs, J.C., et al 1994 Proc. of 21st EPS

[32] David, P., et al 2017 Rev. of Sci. Instruments $\mathbf{8 8 . 1 1} 113507$

[33] Gloggler, S., 2018 Masters Thesis, Max Planck Institute for Plasma Physics, Garching, Germany

[34] Odstrcil, T., et al 2016 Rev. of Sci. Instruments $\mathbf{8 7 . 1 2} 123505$

[35] Bernert, M., 2010 Masters Thesis,Max Planck Institute for Plasma Physics, Garching, Germany

[36] Shiraki, D., et al 2018 60th APS http://meetings.aps.org/link/BAPS.2018.DPP.NP11.115

[37] Shiraki, D., et al 2015 Nucl. Fusion 55.7073029 